\title{
International Normalized Ratio Does Not Predict Gastrointestinal Bleeding After Endoscopic Retrograde Cholangiopancreatography in Patients With Cirrhosis
}

\author{
Abimbola Adikea, c, Mohanad Al-Qaisi ${ }^{\mathrm{a}}$, Noemi J. Baffy ${ }^{\mathrm{a}}$, Heidi Kosiorek ${ }^{\mathrm{b}}$, Rahul Pannala ${ }^{\mathrm{a}}$, \\ Bashar Aqel $^{\mathrm{a}}$, Douglas O. Faigel ${ }^{\mathrm{a}}$, M. Edwyn Harrison ${ }^{\mathrm{a}}$
}

\begin{abstract}
Background: Cirrhosis is often accompanied by an elevated international normalized ratio (INR) due to a decrease in pro-coagulant factors. An elevated INR in cirrhosis is often interpreted as an increased risk of bleeding. There are a paucity of data in the literature on the use of INR to predict risk of gastrointestinal bleeding (GIB) following endoscopic retrograde cholangiopancreatography (ERCP) in patients with cirrhosis. The aims of the study were to determine if there is a correlation between INR and GIB following ERCP in patients with cirrhosis, and to determine if there is a difference in frequency of post-ERCP complications in patients with and without cirrhosis.
\end{abstract}

Methods: A retrospective review of all ERCP procedures was performed at a tertiary care institution between 2012 and 2015. We identified ERCPs performed in patients with cirrhosis and compared them to a randomly selected group without liver cirrhosis. Univariate analysis was performed using Chi-square and ANOVA tests. A multivariable logistic regression model using generalized estimating equations was used to examine the association between INR and GIB.

Results: There were a total of 1,610 ERCPs performed from 2012 to 2015 with 129 performed in 56 patients with cirrhosis compared with 392 ERCPs performed in 310 patients without cirrhosis. There was no difference in the frequency of GIB following ERCP in both groups $(\mathrm{P}=0.117)$. However, there was a difference in overall complications between both groups $(\mathrm{P}=0.007)$, but no difference observed amongst Child-Turcotte-Pugh classes ( $\mathrm{P}=\mathrm{NS})$. In a multivariable analysis, sphincterotomy during ERCP (odds ratio $(\mathrm{OR})=3.22 ; 95 \%$ confidence interval $(\mathrm{CI}): 1.05$ - 9.94; $\mathrm{P}=0.042)$ and cirrhosis $(\mathrm{OR}=3.58$; 95\% CI: 1.22 - 10.47; P = 0.02) were significant for predicting GIB. Anti-coagulation $(\mathrm{OR}=2.90 ; 95 \% \mathrm{CI}: 0.82-10.23 ; \mathrm{P}=0.097)$ and

Manuscript submitted June 4, 2017, accepted June 23, 2017

aDivision of Gastroenterology and Hepatology, Mayo Clinic, Scottsdale, AZ, USA

bDivision of Health Sciences Research, Mayo Clinic, Scottsdale, AZ, USA ${ }^{\mathrm{c} C o r r e s p o n d i n g ~ A u t h o r: ~ A b i m b o l a ~ A d i k e, ~ D i v i s i o n ~ o f ~ G a s t r o e n t e r o l o g y ~ a n d ~}$ Hepatology, Mayo Clinic, 13400 E Shea Blvd, Scottsdale, AZ 85259, USA. Email: adike.abimbola@mayo.edu

doi: https://doi.org/10.14740/gr873w
INR were not significant in the multivariable model $(\mathrm{OR}=2.09 ; 95 \%$ CI: $0.85-5.12 ; \mathrm{P}=0.10$ ).

Conclusion: There was a statistical difference in overall complications between patients with and without cirrhosis but no difference was observed amongst Child-Turcotte-Pugh classes. Overall, INR was not a significant factor in predicting risk of bleeding in patients after ERCP.

Keywords: INR; ERCP; End-stage liver disease; Cirrhosis; Gastrointestinal bleeding

\section{Introduction}

Patients with cirrhosis often have profound abnormalities in the hemostatic system. Coagulation abnormalities and bleeding diathesis are frequently encountered with cirrhosis. These patients exist in a precarious hemostatic rebalance with thrombocytopenia, and abnormal international normalized ratio (INR) or prothrombin time (PT) levels [1-3]. Interpretation of these tests in cirrhosis is unclear as there may be a hypercoagulable or hypocoagulable state $[2,4]$.

It is unknown how the use of INR predicts risk of bleeding following procedures in patients with cirrhosis. Several studies have found a similar complication rate in those with cirrhosis undergoing cardiac procedures compared to those without, and have shown that elevated INR is not associated with an increased bleeding risk [4-6]. In a study by Townsend et al of 240 patients undergoing right and left heart catheterizations, they found that there was no significant difference in procedural complications in those with a normal or elevated INR [4]. There is also little evidence to suggest that fresh frozen plasma (FFP) administered as prophylaxis prior to invasive procedures prevents bleeding complications after invasive procedures $[4,7]$.

Endoscopic retrograde cholangiopancreatography (ERCP) is an important diagnostic and therapeutic tool used for hepatobiliary disease. It is considered one of the highest-risk endoscopic procedures. There are little data on how INR predicts bleeding complications after ERCP [3, 8]. Hence, the primary goal of this study was to determine the relationship between INR and gastrointestinal bleeding (GIB) complications follow- 
Table 1. Univariate Analysis Comparing ERCP Procedures in Cirrhotic Patients vs. Non-Cirrhotic Patients

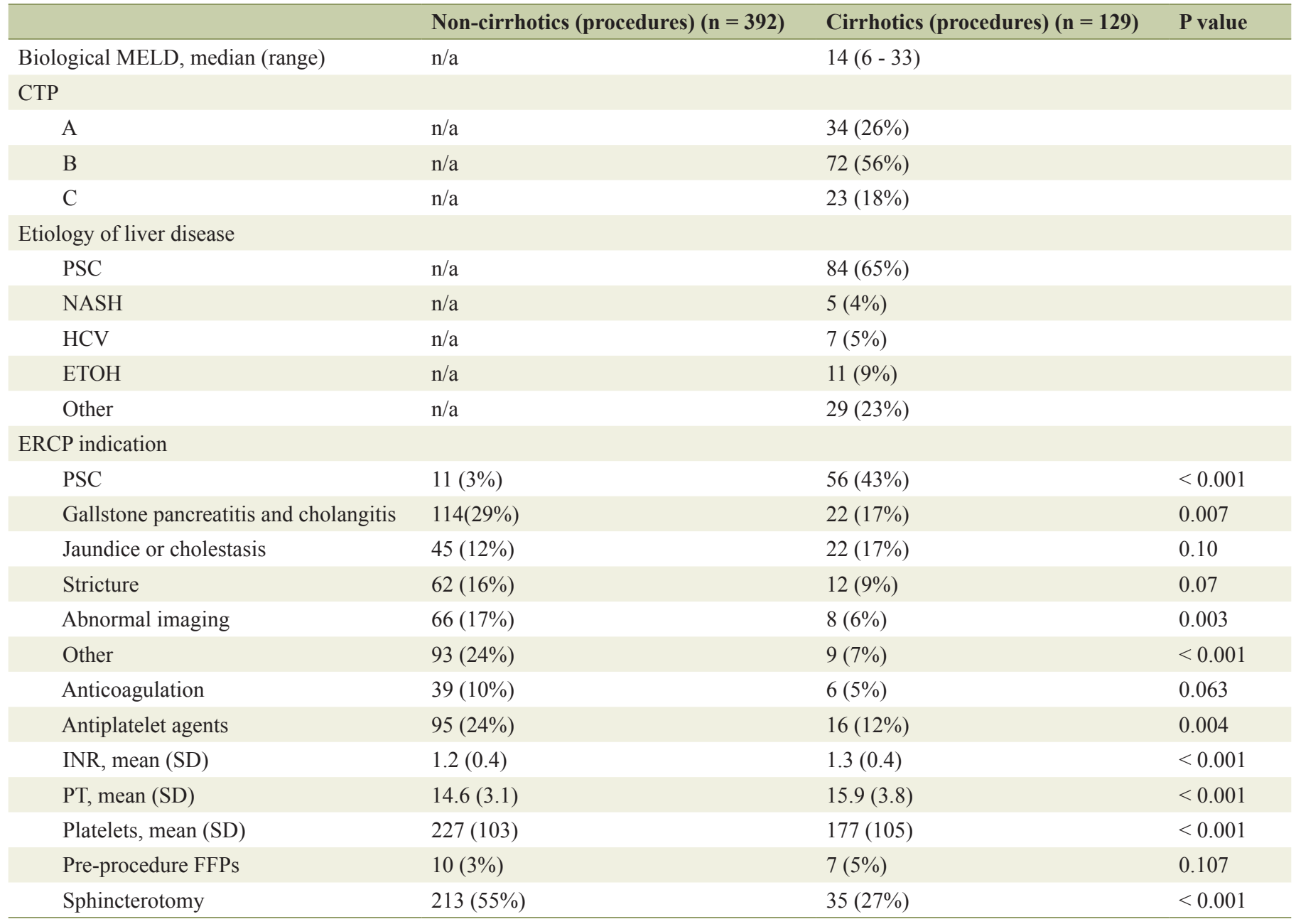

ing ERCP in patients with cirrhosis. In addition, as there have only been a few studies $[9,10]$ that have evaluated complications after ERCP in this group of patients, our secondary goal was to determine the risk of post-ERCP adverse events in patients with cirrhosis.

\section{Methods}

\section{Setting}

A retrospective review of all ERCP procedures was performed at a tertiary care institution between 2012 and 2015 .

\section{Data collection}

We collected demographic information of the patients including age, gender, anticoagulation or antiplatelet therapy at time of procedure, etiology of cirrhosis and indication for ERCP. Patients who had an existing bleeding diathesis (such as im- mune thrombocytopenia (ITP) or disseminated intravascular coagulopathy (DIC)) were also noted. Laboratory values including INR, PT, platelets, hemoglobin, albumin, creatinine, total bilirubin, and sodium, at the time of procedure were collected. We also determined patients who received pre-, periprocedural or post-procedural blood products. MELD scores and Child-Turcotte-Pugh (CTP) classification at time of procedure were determined. Specific interventions performed during the ERCP were collected (sphincterotomy, precut sphincterotomy, extension of sphincterotomy, balloon sweep, balloon dilation or stent placement). All complications (GIB, cholangitis, post-ERCP pancreatitis, perforation, anesthesia-related complications and death) within 30 days after ERCP were documented. Adverse events of cirrhotic patients who underwent ERCP were compared with a control group of randomly selected non-cirrhotic patients.

\section{Data analysis}

Univariate analysis was performed using Chi-square (or 
Table 2. Post-Procedure Complications

\begin{tabular}{llll}
\hline & Non cirrhotics $(\mathbf{n}=\mathbf{3 9 2})$ & Cirrhotics $(\mathbf{n}=\mathbf{1 2 9})$ & P value \\
\hline Any complication & $52(13 \%)$ & $25(19 \%)$ & 0.007 \\
Child-Turcotte-Pugh A & $\mathrm{n} / \mathrm{a}$ & $4(16 \%)$ & $16(64 \%)$ \\
Child-Turcotte-Pugh B & $\mathrm{n} / \mathrm{a}$ & $5(20 \%)$ & 0.117 \\
Child-Turcotte-Pugh C & $\mathrm{n} / \mathrm{a}$ & $8(6 \%)$ & 0.025 \\
GI bleeding & $12(3 \%)$ & $5(4 \%)$ & 0.630 \\
GI bleeding requiring blood transfusion & $3(0.1 \%)$ & $7(5 \%)$ & 0.015 \\
Post-ERCP pancreatitis & $17(4 \%)$ & $8(6 \%)$ & 0.618 \\
Cholangitis & $7(2 \%)$ & $4(3 \%)$ & \\
Death & $17(4 \%)$ & & \\
\hline
\end{tabular}

Fisher's exact test) and ANOVA tests. A multivariable logistic regression model using generalized estimating equations was used to examine the association between INR and GIB complications post-ERCP, as well as risk factors for increased complications post-ERCP.

\section{Results}

There were a total of 1,610 ERCPs performed from 2012 to 2015 with 129 performed in 56 patients with cirrhosis compared with 392 ERCPs performed in 310 patients without cirrhosis. The mean age of patients without cirrhosis was 63.1 years $( \pm 14.9)$, while the mean age of patients with cirrhosis was 58.8 years $( \pm 14.6)(\mathrm{P}=0.005)$. Fifty-two percent of patients without cirrhosis and $59 \%$ of patients with cirrhosis were males $(\mathrm{P}=0.33)$. Median biological MELD score in patients with cirrhosis was 14 (range 6 - 33), with majority of patients with a Child-Turcotte-Pugh (CTP) classification of B (56\%). The most common etiology of end-stage liver disease was primary sclerosing cholangitis (PSC) $(65 \%)$. PSC was the most common indication for ERCP (43\%) in patients with cirrhosis. In patients without cirrhosis, the most common indications for ERCP were cholangitis or gallstone pancreatitis (29\%) and other $(24 \%)$, which included pancreaticobiliary malignancy, abnormal liver enzyme tests, chronic and recurrent acute pancreatitis, bile leak, and abdominal pain with suspected sphincter of Oddi dysfunction (Table 1).

There were more patients without cirrhosis on anticoagula-

Table 3. Characteristics by GI Bleeding in All Patients

\begin{tabular}{llll} 
& No GIB (n= 501) & GIB (n= 20) & P value \\
\hline Anticoagulation & $40(8 \%)$ & $5(25 \%)$ & 0.008 \\
Antiplatelet & $107(21 \%)$ & $4(20 \%)$ & 0.884 \\
Pre-procedure FFPs & $13(3 \%)$ & $4(20 \%)$ & 0.003 \\
Sphincterotomy & $234(47 \%)$ & $14(70 \%)$ & 0.065 \\
INR, mean (SD) & $1.2(0.3)$ & $1.5(0.8)$ & $<0.001$ \\
PT, mean (SD) & $15(3)$ & $18(7)$ & 0.001 \\
Platelet, mean (SD) & $215(106)$ & $206(85)$ & 0.828 \\
\hline
\end{tabular}

tion and antiplatelet agents than there were in the group with cirrhosis (Table 1). There was a significant difference in the INR and platelet count between both groups. Patients with cirrhosis received more pre-procedural FFP products than those without cirrhosis $(\mathrm{P}=\mathrm{NS})$. Sphincterotomy was more commonly performed in the group without cirrhosis $(\mathrm{P}<0.001)$, (Table 1$)$. There was no difference in the frequency of GIB following ERCP in both groups $(\mathrm{P}=0.117)$ but more patients in the cirrhotic group required blood transfusions compared to the noncirrhotic group $(\mathrm{P}=0.025)$, (Table 2$)$. There was a difference in overall complications between both groups $(\mathrm{P}=0.007)$ with a significant difference observed in the rates of post-ERCP cholangitis between both groups $(\mathrm{P}=0.015)$, (Table 2$)$. In cirrhotic patients, there was no statistical difference observed between CTP classes for complications. Amongst those who had GIB in cirrhotic patients, the range of INR was $1.06-4.53$, and in noncirrhotic patients with GIB, the range of INR was $0.91-2.1$.

On univariate analysis in all patients, sphincterotomy, platelets, INR and PT were important risk factors for GIB (Table 3). On univariate analysis in cirrhotic patients only, INR was not significantly different between patients with and without GIB (Table 4). On multivariable analysis, sphincterotomy during $\mathrm{ERCP}(\mathrm{OR}=3.22 ; 95 \%$ confidence interval $(\mathrm{CI}): 1.05$ - 9.94; $\mathrm{P}=0.042)$ and cirrhosis $(\mathrm{OR}=3.58 ; 95 \% \mathrm{CI}: 1.22$ - 10.47; P $=0.02$ ) were significant for predicting GIB. Anticoagulation $(\mathrm{OR}=2.90 ; 95 \% \mathrm{CI}: 0.82-10.23 ; \mathrm{P}=0.097)$ and INR were not significant in the multivariable model $(\mathrm{OR}=$ 2.09; 95\% CI: $0.85-5.12 ; \mathrm{P}=0.10)$. Age and gender were not significant in the multivariate analysis.

Table 4. Characteristics by GI Bleeding in Cirrhotic Patients

\begin{tabular}{llll}
\hline & No GIB $(\mathbf{n = 1 2 1})$ & GIB $(\mathbf{n = 8})$ & P value \\
\hline Anticoagulation & $5(4 \%)$ & $1(13 \%)$ & 0.324 \\
Antiplatelet & $16(13 \%)$ & $0(0 \%)$ & 0.272 \\
Pre-procedure FFPs & $5(4 \%)$ & $2(25 \%)$ & 0.060 \\
Sphincterotomy & $30(25 \%)$ & $5(63 \%)$ & 0.034 \\
INR, mean (SD) & $1.2(0.3)$ & $1.7(1.2)$ & 0.257 \\
PT, mean (SD) & $15.7(3.1)$ & $19.3(9.4)$ & 0.401 \\
Platelet, mean (SD) & $176.9(104.3)$ & $180.8(118.1)$ & 0.864 \\
\hline
\end{tabular}




\section{Discussion}

In this study, patients with cirrhosis had higher INR and PT levels even though there were more patients on anticoagulation in the group without cirrhosis. There was no statistical difference in the frequency of GIB between both groups. There was no difference in the frequency of this complication amongst CTP classes. Although the INR was statistically different in patients with or without GIB (Table 3), no difference was observed in INR level between patients with and without GIB when evaluated in a multivariable model.

Patients with cirrhosis have anticoagulant and procoagulant proteins in reduced levels which lead to a precarious rebalanced hemostatic state that may easily shift between hypercoagulability or hypocoagulability $[1,2,11]$. Stravitz et al in their study on acute liver injury using thromboelastography (which uses a different assay than PT/INR assays) showed that this hemostatic rebalance in patients with liver failure is generally persevered in these patients despite an elevated INR [11]. These authors postulate that a commensurate decrease in anticoagulant and procoagulant proteins in liver failure is likely responsible for this observation.

Our findings support the current literature that INR is not a reliable measure of the state of hemostasis in patients with cirrhosis $[2-4,8]$. Pillarisetti et al found that there was no difference in patients with cirrhosis in terms of outcomes of GIB or length of hospital stay after elective cardiac catheterization when compared to patients without cirrhosis, although the authors note that patients with an INR $\geq 1.6$ received FFPs [6]. Townsend et al also found that an elevated INR did not accurately predict bleeding complications after left or right heart catheterization [4]. A systematic review to determine if INR or PT predicts risk of bleeding during an invasive procedure concluded that there is no evidence to show that an elevated INR or PT is predictive of peri-procedural bleeding [8]. Furthermore, there is little evidence that correction of INR elevation with transfusion of FFPs makes any difference in reducing risk of bleeding $[4-6,8,12]$. In a split retrospective and prospective study by Youssef and colleagues, many patients in this study failed to achieve clinically significant change in the prothrombin time after receiving FFPs using the number of units commonly used in clinical practice [7].

Although there was no statistical difference in the frequency of GIB in the cirrhosis group compared to the non-cirrhosis group, patients in the cirrhosis group required more blood transfusion during GIB $(\mathrm{P}=0.025)$. In addition, there was a statistical difference in the frequency of cholangitis post-ERCP in the cirrhosis group, which we feel is largely related to our patient population, with PSC being the primary indication for ERCP in $43 \%$ of patients with cirrhosis. In the study by Adler et al, of 328 patients with cirrhosis, all patients were preoperatively administered vitamin K or FFP if INR was greater than 1.5, and ERCP was avoided if INR $>1.7$ [10]. The authors, nevertheless, report they found that there was no correlation between significant hemorrhage and presence of coagulopathy or CTP class amongst cirrhotic patients. In our study, we also found no significant difference in the frequency of post-ERCP complications amongst CTP class. Other studies have also con- firmed that there is no significant difference found between post-sphincterotomy complications and CTP class [13, 14]. However, adverse events were more notably severe when they did occur in Child class $C$ patients [14]. There have been other studies that have found higher risks of hemorrhage in CTP class $\mathrm{C}$ than in other cirrhotics [15]. In addition, although Adler et al found no difference in the adverse events amongst CTP class A, $\mathrm{B}$ and $\mathrm{C}(\mathrm{P}=0.068), \mathrm{CTP}$ class $\mathrm{B}$ and $\mathrm{C}$ had a higher incidence of adverse events when compared with CTP class A $(\mathrm{P}=0.048)$ [10]. Adler et al also found that patients with encephalopathy had a higher risk of adverse events [10]. Decompensated cirrhosis as a risk factor in predicting complications post-ERCP is further corroborated by the study by Inamdar et al who found no difference in patients with compensated cirrhosis vs. non-cirrhotic controls (except for post-ERCP pancreatitis) but found a statistically significant difference in the rate of GIB and post-ERCP pancreatitis between patients with decompensated cirrhosis and non-cirrhotic controls [16]. However, like Navaneethan et al [9], we also found that cirrhosis was significant in predicting risk of GIB following ERCP and there was no difference between Child-Pugh classes.

There are several limitations of our study, inherent in its retrospective nature. Patients may have had other complications following ERCP and may have presented at a health facility other than our institution, and thus, these potential complications may have been missed by this study. Selection bias may also be present in that the endoscopist may be more cautious in avoiding sphincterotomy when a patient is known to have cirrhosis, and this is reflected in the significant difference in the number of patients with cirrhosis who underwent sphincterotomy versus those without cirrhosis. In addition, although we randomly selected non-cirrhotic patients, this was an unmatched group and so there may be co-existing conditions predisposing them to complications which are not accounted for in our study. In addition, this is a single-center study at a tertiary institution and our results may not be generalizable to other centers. Despite these limitations, our study has several strengths. Importantly, no prior study has analyzed use of INR to predict GIB after ERCP in patients with or without cirrhosis. The post-procedure complication rates we report are similar to what have previously been reported. We found that there was a statistical difference in overall complications between both groups with no difference observed amongst CTP classes.

In summary, in concurrence with recent literature showing that INR is not a useful indicator of the hemostatic state in cirrhosis, we conclude that an elevated INR is not a significant factor in predicting risk of post-ERCP GIB.

\section{Author Contributions}

Abimbola Adike, Mohanad Al-Qaisi, and Noemi J. Baffy collected the data and collectively wrote the manuscript. Heid Kosiorek analyzed the data. Rahul Pannala, Bashar Aqel, Douglas O. Faigel, and M. Edwyn Harrison critically edited the manuscript and made significant contributions to the content of the manuscript. M. Edwyn Harrison developed the original idea for the project, provided overall guidance, and edited the final 
version of the manuscript.

\section{Financial Support}

None.

\section{Conflicts of Interest}

None.

\section{References}

1. Cerini F, Gonzalez JM, Torres F, Puente A, Casas M, Vinaixa $\mathrm{C}$, Berenguer $\mathrm{M}$, et al. Impact of anticoagulation on upper-gastrointestinal bleeding in cirrhosis. A retrospective multicenter study. Hepatology. 2015;62(2):575583.

2. Lisman T, Caldwell SH, Burroughs AK, Northup PG, Senzolo M, Stravitz RT, Tripodi A, et al. Hemostasis and thrombosis in patients with liver disease: the ups and downs. J Hepatol. 2010;53(2):362-371.

3. Krzanicki D, Sugavanam A, Mallett S. Intraoperative hypercoagulability during liver transplantation as demonstrated by thromboelastography. Liver Transpl. 2013;19(8):852-861.

4. Townsend JC, Heard R, Powers ER, Reuben A. Usefulness of international normalized ratio to predict bleeding complications in patients with end-stage liver disease who undergo cardiac catheterization. Am J Cardiol. 2012;110(7):1062-1065.

5. Mahmoud AM, Elgendy IY, Choi CY, Bavry AA. Risk of bleeding in end-stage liver disease patients undergoing cardiac catheterization. Tex Heart Inst J. 2015;42(5):414418.

6. Pillarisetti J, Patel P, Duthuluru S, Roberts J, Chen W, Genton R, Wiley M, et al. Cardiac catheterization in patients with end-stage liver disease: safety and outcomes. Catheter Cardiovasc Interv. 2011;77(1):45-48.

7. Youssef WI, Salazar F, Dasarathy S, Beddow T, Mullen $\mathrm{KD}$. Role of fresh frozen plasma infusion in correction of coagulopathy of chronic liver disease: a dual phase study. Am J Gastroenterol. 2003;98(6):1391-1394.

8. Segal JB, Dzik WH, Transfusion Medicine/Hemostasis Clinical Trials N. Paucity of studies to support that abnormal coagulation test results predict bleeding in the setting of invasive procedures: an evidence-based review. Transfusion. 2005;45(9):1413-1425.

9. Navaneethan U, Njei B, Zhu X, Kommaraju K, Parsi MA, Varadarajulu S. Safety of ERCP in patients with liver cirrhosis: a national database study. Endosc Int Open. 2017;5(4):E303-E314.

10. Adler DG, Haseeb A, Francis G, Kistler CA, Kaplan J, Ghumman SS, Laique SN, et al. Efficacy and safety of therapeutic ERCP in patients with cirrhosis: a large multicenter study. Gastrointest Endosc. 2016;83(2):353359.

11. Stravitz RT, Lisman T, Luketic VA, Sterling RK, Puri P, Fuchs M, Ibrahim A, et al. Minimal effects of acute liver injury/acute liver failure on hemostasis as assessed by thromboelastography. J Hepatol. 2012;56(1):129-136.

12. Shah NL, Northup PG, Caldwell SH. A clinical survey of bleeding, thrombosis, and blood product use in decompensated cirrhosis patients. Ann Hepatol. 2012;11(5):686690.

13. Zhang J, Ye L, Zhang J, Lin M, He S, Mao X, Zhou X, et al. MELD scores and Child-Pugh classifications predict the outcomes of ERCP in cirrhotic patients with choledocholithiasis: a retrospective cohort study. Medicine (Baltimore). 2015;94(3):e433.

14. Prat F, Tennenbaum R, Ponsot P, Altman C, Pelletier G, Fritsch J, Choury AD, et al. Endoscopic sphincterotomy in patients with liver cirrhosis. Gastrointest Endosc. 1996;43(2 Pt 1):127-131.

15. Li DM, Zhao J, Zhao Q, Qin H, Wang B, Li RX, Zhang $M$, et al. Safety and efficacy of endoscopic retrograde cholangiopancreatography for common bile duct stones in liver cirrhotic patients. J Huazhong Univ Sci Technolog Med Sci. 2014;34(4):612-615.

16. Inamdar S, Berzin TM, Berkowitz J, Sejpal DV, Sawhney MS, Chutanni R, Pleskow DK, et al. Decompensated cirrhosis may be a risk factor for adverse events in endoscopic retrograde cholangiopancreatography. Liver Int. 2016;36(10):1457-1463. 